G. Benke and D.-C. Chang

Nagoya Math. J.

Vol. 159 (2000), 25-43

\title{
A NOTE ON WEIGHTED BERGMAN SPACES AND THE CESÁRO OPERATOR
}

\author{
GEORGE BENKE AND DER-CHEN CHANG*
}

Dedicated to Professor John Benedetto

\begin{abstract}
Let $B$ denote the unit ball in $\mathbb{C}^{n}$, and $d V(z)$ normalized Lebesgue measure on $B$. For $\alpha>-1$, define $d V^{\alpha}(z)=\left(1-|z|^{2}\right)^{\alpha} d V(z)$. Let $\mathcal{H}(B)$ denote the space of holomorhic functions on $B$, and for $0<p<\infty$, let $\mathcal{A}^{p}\left(d V_{\alpha}\right)$ denote $L^{p}\left(d V_{\alpha}\right) \cap \mathcal{H}(B)$. In this note we characterize $\mathcal{A}^{p}\left(d V_{\alpha}\right)$ as those functions in $\mathcal{H}(B)$ whose images under the action of a certain set of differential operators lie in $L^{p}\left(d V_{\alpha}\right)$. This is valid for $1 \leq p<\infty$. We also show that the Cesàro operator is bounded on $\mathcal{A}^{p}\left(d V_{\alpha}\right)$ for $0<p<\infty$. Analogous results are given for the polydisc.
\end{abstract}

\section{$\S 0$. Introduction}

Let $B=\left\{z=\left(z_{1}, \ldots, z_{n}\right) \in \mathbb{C}^{n}:|z|<1\right\}$ be the unit ball in $\mathbb{C}^{n}$, let $\mathcal{H}(B)$ be the class of all holomorphic functions defined on $B$, and let $d V^{\alpha}(z)=\left(1-|z|^{2}\right)^{\alpha} d V(z)$ where $d V(z)$ is Lebesgue measure on $B$ normalized to make the volume of the unit ball equal 1 , i.e,

$$
\int_{B} d V(z)=\frac{n \Gamma(n)}{\pi^{n}} \int_{0}^{1} \int_{\partial B} r^{2 n-1} d r d \sigma=1
$$

(see $[\mathrm{K}$, page 58]). We are interested in the holomorhpic functions which lie in $L^{p}\left(d V_{\alpha}\right)$ for various $0<p<\infty$ and $\alpha>-1$. The case $p=2$ and $\alpha=0$ involves the classical Bergman projection operator - one of the most important operators in the theory of functions of several complex variables. It has been used to characterize biholomorphic mappings of finite type

Received August 31, 1998.

Revised July 12, 1999.

2000 Mathematics Subject Classification: Primary: 32A25, Secondary: 32A36, 65B10.

*The research is partially supported by a grant from the National Science Foundation. The second author is also partially supported by a William Fulbright Scholarship and a competative research grant at Georgetown University 
pseudoconvex domains (see Fefferman [F], Bell and Ligocka [BL], Catlin $[\mathrm{Ca}])$.

It is worth noting that $d V_{\alpha}(z)$ is a natural measure on $B$ since the projection operator from $\mathbb{C}^{n}$ onto $\mathbb{C}^{m}, 1 \leq m<n$, restricted to the sphere in $\mathbb{C}^{n}$ is naturally expressed as integration with respect to $d V_{\alpha}(z)$ over the unit ball $B_{m}$ in $\mathbb{C}^{m}$ with $\alpha=n-m-1$. More specifically, Forelli [Fo] has shown that

$$
\begin{aligned}
\int_{\partial B} & (f \circ \mathbf{P}) d \sigma \\
& =\frac{(n-1) !}{m !(n-m-1) !} \int_{B_{m}}\left(1-\sum_{k=1}^{m}\left|z_{k}\right|^{2}\right)^{n-m-1} f\left(z_{1}, \ldots, z_{m}\right) d V_{m}(z) .
\end{aligned}
$$

where $d \sigma$ is the area measure on $\partial B$ and $d V_{m}$ is the Lebesgue measure on $\mathbb{C}^{m}$.

We define $\mathcal{A}^{p}\left(d V_{\alpha}\right)$ to be the intersection of the spaces $L^{p}\left(d V_{\alpha}\right)$ and $\mathcal{H}(B)$, and call this the weighted Bergman space. It turns out that $\mathcal{A}^{p}\left(d V_{\alpha}\right)$ is a closed subspace of $L^{p}\left(d V_{\alpha}\right)$ and so it is natural to consider the projection

$$
\mathbf{B}_{\alpha}: L^{p}\left(d V_{\alpha}\right) \rightarrow \mathcal{A}^{p}\left(d V_{\alpha}\right)
$$

This projection, known as the weighted Bergman projection, is given an integral over $B$ and is known as the weighted Bergman integral on $B$. The object of this paper is to give another characterization of $\mathcal{A}^{p}\left(d V_{\alpha}\right)$, namely, that $\mathcal{A}^{p}\left(d V_{\alpha}\right)$ consists of those holomorphic functions whose images under a certain set of differential operators lie in $L^{p}\left(d V_{\alpha}\right)$. This is the content of Theorem 1.7. We also observe in Theorem 1.8 that a similar characterization exists when the ball is replaced by the polydisc. A second objective of the paper is to study the Cesàro operator on $\mathcal{A}^{p}\left(d V_{\alpha}\right)$, for both the ball and the polydisc. Here we prove that in both of these cases the Cesàro operator is a bounded operator. This is the content of Theorems 2.4 and 2.5.

The paper is organized as follows. In section 1 we record the relevant definitions and lemmas, omitting proofs when they are available elsewhere in the literature. The main Theorems 1.7 and 1.8, characterizing $\mathcal{A}^{p}\left(d V_{\alpha}\right)$ are proven. In section 2 we define the Cesàro operator for the polydisc and the "slice" Cesàro operator for the ball. In Theorem 2.4 we prove that the Cesàro operator is a bounded operator on $\mathcal{A}^{p}\left(d V_{\alpha}\right)$ for the polydisc. We state the corresponding result for the slice Cesàro operator on the ball. The proof is omitted since it is similar to the case of the polydisc. 
The authors would like to thank the referee for many valuable suggestions.

\section{$\S 1$. Weighted Bergman space}

The space $L^{p}\left(d V_{\alpha}\right)$ consists of all Lebesgue measurable functions $f$ defined on $B$ satisfying

$$
\|f\|_{L^{p}\left(d V_{\alpha}\right)}^{p}=\int_{B}|f(z)|^{p}\left(1-|z|^{2}\right)^{\alpha} d V(z)<\infty
$$

where $d V(z)$ is the volume measure on $B$ normalized so that $V(B)=1$. It is easy to see that for $1 \leq p<\infty, L^{p}\left(d V_{\alpha}\right)$ is a Banach space with norm $\|\cdot\|_{L^{p}\left(d V_{\alpha}\right)}$ and for $0<p<1, L^{p}\left(d V_{\alpha}\right)$ is an F-space under the metric

$$
d(f, g)=\|f-g\|_{L^{p}\left(d V_{\alpha}\right)}^{p} .
$$

Definition. For $0<p<\infty$, and $\alpha>-1$

$$
\mathcal{A}^{p}\left(d V_{\alpha}\right)=L^{p}\left(d V_{\alpha}\right) \cap \mathcal{H}(B)
$$

The following lemma is proved, for example, in Djrbashian and Shamoian [DS], page 14, Corollary 1 to Theorem 1.1 and pages $128-136$, $\S 6.1$.

Lemma 1.1. For $1 \leq p<\infty, \mathcal{A}^{p}\left(d V_{\alpha}\right)$ is a closed subspace of $L^{p}\left(d V_{\alpha}\right)$.

For $p=2$ we define the weighted Bergman projection

$$
\mathbf{B}_{\alpha}: L^{2}\left(d V_{\alpha}\right) \rightarrow \mathcal{A}^{2}\left(d V_{\alpha}\right), \quad \alpha>-1
$$

as follows:

$$
\begin{aligned}
& \mathbf{B}_{\alpha}(f)(z) \\
= & \frac{(\alpha+1) \cdots(\alpha+n)}{n !} \int_{B} \frac{f(w)}{(1-z \cdot \bar{w})^{n+1+\alpha}}\left(1-|w|^{2}\right)^{\alpha} d V(w) \\
= & \int_{B} f(w) K_{\alpha}(z, w)\left(1-|w|^{2}\right)^{\alpha} d V(w) .
\end{aligned}
$$

The following result allows the extention to other values of $p$. 
Proposition 1.2. Let $-1<\alpha<\infty,-1<\beta<\infty$ and $1<p<\infty$. Then the projection operator $\mathbf{B}_{\beta}$ can be extended as a bounded operator from $L^{p}\left(d V_{\alpha}\right)$ onto $\mathcal{A}^{p}\left(d V_{\alpha}\right)$ if and only if $(1+\beta) p>1+\alpha$. Moreover, in this case we have the reproducing formula $\mathbf{B}_{\beta}(f)=f$ for all $f \in \mathcal{A}^{p}\left(d V_{\alpha}\right)$.

For the proof, see [DS, pages 33-36], § 2.1 and pages 128-136. We also refer the readers to $[\mathrm{BG}],[\mathrm{BCG}],[\mathrm{CL}]$ and references in there for further discussions.

Remark. Consider the case $\beta=\alpha$ in the hypothesis of Proposition 1.2 , it is obvious that the operator $\mathbf{B}_{\alpha}$ is not bounded on $L^{1}\left(d V_{\alpha}\right)$ (see also [CNS], [CL]). However, for $\alpha>-1$, we may consider the following projection operator:

$$
\begin{aligned}
\tilde{\mathbf{B}}_{\alpha}(f)(z)=\frac{(\alpha+2) \cdots(\alpha+n+1)}{n !} \times \\
\times \int_{B} \frac{f(w)}{(1-z \cdot \bar{w})^{n+2+\alpha}}\left(1-|w|^{2}\right)^{\alpha+1} d V(w) .
\end{aligned}
$$

Then it can be shown that $\tilde{\mathbf{B}}_{\alpha}: L^{1}\left(d V_{\alpha}\right) \rightarrow \mathcal{A}^{1}\left(d V_{\alpha}\right)$ boundedly. Moreover, $\tilde{\mathbf{B}}_{\alpha}(f)=f$ for all $f \in \mathcal{A}^{1}\left(d V_{\alpha}\right)$ (see e.g., [Z, Chapter 4]). Proofs for the results in this section for the operator $\tilde{\mathbf{B}}_{\alpha}$ are identical to the proofs of the corresponding results for the operator $\mathbf{B}_{\alpha}$. Therefore, we will not repeat them.

Let $\mathbb{Z}_{+}=\{0,1,2, \cdots\}$. A multi-index $\mathbf{k}=\left(k_{1}, \ldots, k_{n}\right)$ is an element in $\left(\mathbb{Z}_{+}\right)^{n}$. If $\mathbf{k}$ is a multi-index, let $|\mathbf{k}|=k_{1}+\cdots+k_{n}$ and define the operator $\mathcal{Q}_{\mathbf{k}}$ as follows:

$$
\mathcal{Q}_{\mathbf{k}}(f)(z)=\left(1-|z|^{2}\right)^{|\mathbf{k}|} \int_{B} \frac{\bar{w}_{1}^{k_{1}} \cdots \bar{w}_{n}^{k_{n}} f(w)}{(1-z \cdot \bar{w})^{n+1+\alpha+|\mathbf{k}|}}\left(1-|w|^{2}\right)^{\alpha} d V(w) .
$$

Lemma 1.3. Let $1<p<\infty$ and $\alpha>-1$. Then the operator $\mathcal{Q}_{\mathbf{k}}$ can be extended as a bounded operator from $L^{p}\left(d V_{\alpha}\right)$ into $L^{p}\left(d V_{\alpha}\right)$.

Proof. Let $q$ be the conjugate exponent of $p$, i.e., $\frac{1}{p}+\frac{1}{q}=1$. Since $w \in B,\left|\bar{w}_{1}^{k_{1}} \cdots \bar{w}_{n}^{k_{n}}\right| \leq 1$, we know that

$$
\begin{aligned}
& \left|\mathcal{Q}_{\mathbf{k}}(f)(z)\right|^{p} \\
& \leq\left\{\left(1-|z|^{2}\right)^{|\mathbf{k}|} \int_{B} \frac{|f(w)|\left(1-|w|^{2}\right)^{-\frac{\alpha+1}{2 p q}}\left(1-|w|^{2}\right)^{\frac{\alpha+1}{2 p q}}}{|1-z \cdot \bar{w}|^{n+1+\alpha+|\mathbf{k}|}}\left(1-|w|^{2}\right)^{\alpha} d V(w)\right\}^{p} .
\end{aligned}
$$


Then by Hölder's inequality, we have

$$
\begin{aligned}
& \left|\mathcal{Q}_{\mathbf{k}}(f)(z)\right|^{p} \leq\left(1-|z|^{2}\right)^{p|\mathbf{k}|}\left\{\int_{B} \frac{\left(1-|w|^{2}\right)^{-\frac{\alpha+1}{2 p}}}{|1-z \cdot \bar{w}|^{n+1+\alpha}}\left(1-|w|^{2}\right)^{\alpha} d V(w)\right\}^{\frac{p}{q}} \\
& \times\left\{\int_{B} \frac{|f(w)|^{p}\left(1-|w|^{2}\right)^{\frac{\alpha+1}{2 q}}}{|1-z \cdot \bar{w}|^{n+1+\alpha+p|\mathbf{k}|}}\left(1-|w|^{2}\right)^{\alpha} d V(w)\right\} \\
& \leq C \cdot\left(1-|z|^{2}\right)^{p|\mathbf{k}|-\frac{\alpha+1}{2 \mathbf{q}}}\left\{\int_{B} \frac{|f(w)|^{p}\left(1-|w|^{2}\right)^{\alpha+\frac{\alpha+1}{2 q}}}{|1-z \cdot \bar{w}|^{n+1+\alpha+p|\mathbf{k}|}} d V(w)\right\} \text {. }
\end{aligned}
$$

Since $p|\mathbf{k}|+\alpha-\frac{\alpha+1}{2 q}>-1$, by [R, Proposition 1.4.10], we obtain

$$
\begin{aligned}
& \int_{B}\left|\mathcal{Q}_{\mathbf{k}}(f)(z)\right|^{p}\left(1-|z|^{2}\right)^{\alpha} d V(z) \\
& \leq C \int_{B}\left(1-|w|^{2}\right)^{\alpha+\frac{\alpha+1}{2 q}}|f(w)|^{p}\left\{\int_{B} \frac{\left(1-|z|^{2}\right)^{p|\mathbf{k}|+\alpha-\frac{\alpha+1}{2 q}}}{|1-z \cdot \bar{w}|^{n+1+\alpha+p|\mathbf{k}|}} d V(z)\right\} d V(w) \\
& \leq C \int_{B}|f(w)|^{p}\left(1-|w|^{2}\right)^{\alpha+\frac{\alpha+1}{2 q}-\frac{\alpha+1}{2 q}} d V(w) \leq C \cdot\|f\|_{L^{p}\left(d V_{\alpha}\right)^{p}}^{p}
\end{aligned}
$$

This concludes the proof of Lemma 1.3.

Lemma 1.4. Let $1<p<\infty, \alpha>-1$ and $\mathbf{k} \in\left(\mathbb{Z}_{+}\right)^{n}$. Then $(1-$ $\left.|z|^{2}\right)^{|\mathbf{k}|} \frac{\partial^{\mid} \mathbf{k} \mid f}{\partial z_{1}^{k_{1} \ldots \partial z_{n}^{k_{n}}}}(z) \in L^{p}\left(d V_{\alpha}\right)$ for all $f \in \mathcal{A}^{p}\left(d V_{\alpha}\right)$.

Proof. Since $f \in \mathcal{A}^{p}\left(d V_{\alpha}\right)$, we know that from (1.2),

$$
f(z)=\frac{(\alpha+1) \cdots(\alpha+n)}{n !} \int_{B} \frac{f(w)}{(1-z \cdot \bar{w})^{n+1+\alpha}}\left(1-|w|^{2}\right)^{\alpha} d V(w)
$$

It follows that

$$
\begin{aligned}
\left(1-|z|^{2}\right)^{\mid} \mathbf{k} \mid \frac{\partial^{\mid \mathbf{k}} f}{\partial z_{1}^{k_{1}} \cdots \partial z_{n}^{k_{n}}}(z) & \\
= & \frac{(\alpha+1) \cdots(\alpha+n+|\mathbf{k}|)}{n !}\left(1-|z|^{2}\right)^{\mid} \mathbf{k} \mid \times \\
& \times \int_{B} \frac{\bar{w}_{1}^{k_{1}} \cdots \bar{w}_{n}^{k_{n}} f(w)}{(1-z \cdot \bar{w})^{n+1+\alpha+|\mathbf{k}|}}\left(1-|w|^{2}\right)^{\alpha} d V(w)
\end{aligned}
$$


From Lemma 1.3, there is a constant $C$ depending on $\mathbf{k}, n, \alpha$ and $p$ such that

$$
\left\|\left(1-|z|^{2}\right)^{|\mathbf{k}|} \frac{\partial^{|\mathbf{k}|} f}{\partial z_{1}^{k_{1}} \cdots \partial z_{n}^{k_{n}}}\right\|_{L^{p}\left(d V_{\alpha}\right)} \leq C\|f\|_{\mathcal{A}^{p}\left(d V_{\alpha}\right)} .
$$

Now the result follows immediately.

Similarly, we have the following result for the case $p=1$ (cf. [Z, Lemma 4.2.7]).

Lemma 1.5. Let $\alpha>-1$ and $\mathbf{k} \in\left(\mathbb{Z}_{+}\right)^{n}$. Then

$$
\left(1-|z|^{2}\right)^{|\mathbf{k}|} \frac{\partial^{|\mathbf{k}|} f}{\partial z_{1}^{k_{1}} \cdots \partial z_{n}^{k_{n}}}(z) \in L^{1}\left(d V_{\alpha}\right)
$$

for all $f \in \mathcal{A}^{1}\left(d V_{\alpha}\right)$.

Corollary 1.6. Let $f \in \mathcal{A}^{p}\left(d V_{\alpha}\right)$. Then for all $\mathbf{k} \in\left(\mathbb{Z}_{+}\right)^{n}$

$$
\begin{aligned}
& \frac{\partial^{|\mathbf{k}|} f}{\partial z_{1}^{k_{1}} \cdots \partial z_{n}^{k_{n}}}(0) \\
= & \frac{(\alpha+1) \cdots(\alpha+n+|\mathbf{k}|)}{n !} \int_{B} \bar{w}_{1}^{k_{1}} \cdots \bar{w}_{n}^{k_{n}} f(w)\left(1-|w|^{2}\right)^{\alpha} d V(w) .
\end{aligned}
$$

Furthermore,

$$
\left|\frac{\partial^{|\mathbf{k}|} f}{\partial z_{1}^{k_{1}} \cdots \partial z_{n}^{k_{n}}}(0)\right| \leq C \cdot\|f\|_{\mathcal{A}^{p}\left(d V_{\alpha}\right)} .
$$

Proof. From the equation (1.3), we have the first assertion of the Corollary immediately (cf. [DS, Theorem 6.1]). Now by Hölder's inequality, we obtain

$$
\begin{aligned}
& \left|\frac{\partial^{|\mathbf{k}|} f(0)}{\partial z_{1}^{k_{1}} \cdots \partial z_{n}^{k_{n}}}\right| \\
= & \left|\frac{(\alpha+1) \cdots(\alpha+n+|\mathbf{k}|)}{n !} \int_{B} \bar{w}_{1}^{k_{1}} \cdots \bar{w}_{n}^{k_{n}} f(w)\left(1-|w|^{2}\right)^{\alpha} d V(w)\right| \\
\leq & \frac{(\alpha+1) \cdots(\alpha+n+|\mathbf{k}|)}{n !} \int_{B}|f(w)|\left(1-|w|^{2}\right)^{\alpha} d V(w)
\end{aligned}
$$




$$
\begin{aligned}
& \leq \frac{(\alpha+1) \cdots(\alpha+n+|\mathbf{k}|)}{n !} \cdot\|f\|_{\mathcal{A}^{p}\left(d V_{\alpha}\right)} \cdot\left[\int_{B}\left(1-|w|^{2}\right)^{\alpha} d V(w)\right]^{\frac{1}{q}} \\
& \leq C(\alpha,|\mathbf{k}|, p) \cdot\|f\|_{\mathcal{A}^{p}\left(d V_{\alpha}\right)}
\end{aligned}
$$

where $\frac{1}{p}+\frac{1}{q}=1$.

Now we are in a position to prove the main theorem of this section.

THEOREM 1.7. Let $1 \leq p<\infty, \alpha>-1, N$ be a fixed positive integer and $\mathbf{k}=\left(k_{1}, \ldots, k_{n}\right) \in\left(\mathbb{Z}_{+}\right)^{n}$. Let $f$ be a holomorphic function defined on the unit ball $B$ in $\mathbb{C}^{n}$. Then $f \in \mathcal{A}^{p}\left(d V_{\alpha}\right)$ if and only if

$$
\left(1-|z|^{2}\right)^{N} \frac{\partial^{N} f}{\partial z_{1}^{k_{1}} \cdots \partial z_{n}^{k_{n}}}(z) \in L^{p}\left(d V_{\alpha}\right), \quad \text { for all }|\mathbf{k}|=N
$$

Moreover,

$$
\|f\|_{L^{p}\left(d V_{\alpha}\right)}
$$

$$
\approx\left(\sum_{|\mathbf{k}|=0}^{N-1}\left|\frac{\partial^{|\mathbf{k}|} f}{\partial z_{1}^{k_{1}} \cdots \partial z_{n}^{k_{n}}}(0)\right|+\sum_{|\mathbf{k}|=N}\left\|\left(1-|z|^{2}\right)^{N} \frac{\partial^{N} f}{\partial z_{1}^{k_{1}} \cdots \partial z_{n}^{k_{n}}}\right\|_{L^{p}\left(d V_{\alpha}\right)}\right)
$$

Proof. One direction has been proved in Lemmas 1.4 and 1.5. Now let us turn to the other direction. Assume that

$$
\sum_{|\mathbf{k}|=N}\left(1-|z|^{2}\right)^{N}\left|\frac{\partial^{N} f}{\partial z_{1}^{k_{1}} \cdots \partial z_{n}^{k_{n}}}(z)\right| \in L^{p}\left(d V_{\alpha}\right)
$$

Without loss of generality, we may assume $\frac{\partial^{|\mathbf{k}|} f}{\partial z_{1}^{k_{1}} \ldots \partial z_{n}^{k_{n}}}(0)=0$ for $|\mathbf{k}| \leq 2 N$.

Fix $\mathbf{k} \in\left(\mathbb{Z}_{+}\right)^{n}$ with $|\mathbf{k}|=N$. Now let us consider the function

$$
g(z)=\frac{\left(1-|z|^{2}\right)^{N}}{\bar{z}_{1}^{k_{1}} \cdots \bar{z}_{n}^{k_{n}}} \frac{\partial^{N} f}{\partial z_{1}^{k_{1}} \cdots \partial z_{n}^{k_{n}}}(z)
$$

Then it holds that $g(z) \in L^{p}\left(d V_{\alpha}\right)$. Therefore,

$$
\begin{aligned}
& G(z)=\frac{(\alpha+1) \cdots(\alpha+n)}{n !} \times \\
& \times \int_{B} \frac{\left(1-|w|^{2}\right)^{N}\left(1-|w|^{2}\right)^{\alpha}}{\bar{w}_{1}^{k_{1}} \cdots \bar{w}_{n}^{k_{n}}(1-z \cdot \bar{w})^{n+1+\alpha}} \frac{\partial^{N} f}{\partial w_{1}^{k_{1}} \cdots \partial w_{n}^{k_{n}}}(w) d V(w)
\end{aligned}
$$


is a function in $\mathcal{A}^{p}\left(d V_{\alpha}\right)$. It follows that,

$$
\begin{aligned}
\frac{\partial^{N} G}{\partial z_{1}^{k_{1}} \cdots \partial z_{n}^{k_{n}}}(z)= & \frac{(\alpha+1) \cdots(\alpha+n+N)}{n !} \times \\
& \times \int_{B} \frac{\left(1-|w|^{2}\right)^{N}\left(1-|w|^{2}\right)^{\alpha}}{(1-z \cdot \bar{w})^{n+1+\alpha+N}} \frac{\partial^{N} f}{\partial w_{1}^{k_{1}} \cdots \partial w_{n}^{k_{n}}}(w) d V(w) .
\end{aligned}
$$

Now by Proposition 1.2, we know that

$$
\frac{\partial^{N} G}{\partial z_{1}^{k_{1}} \cdots \partial z_{n}^{k_{n}}}(z)=\frac{\partial^{N} f}{\partial z_{1}^{k_{1}} \cdots \partial z_{n}^{k_{n}}}(z)
$$

for all $z \in B$. For $0 \leq|\mathbf{j}| \leq N-1$,

$$
\begin{aligned}
\frac{\partial^{\mid \mathbf{j}} \mid G}{\partial z_{1}^{j_{1}} \cdots \partial z_{n}^{j_{n}}}(0) & =\frac{(\alpha+1) \cdots(\alpha+n+|\mathbf{j}|)}{n} \times \\
& \times \int_{B} \frac{\left(1-|w|^{2}\right)^{N}\left(1-|w|^{2}\right)^{\alpha}}{\bar{w}_{1}^{k_{1}-j_{1}} \cdots \bar{w}_{n}^{k_{n}-j_{n}}} \frac{\partial^{N} f}{\partial w_{1}^{k_{1}} \cdots \partial w_{n}^{k_{n}}}(w) d V(w)=0 .
\end{aligned}
$$

Thus we have $f(z)=G(z)=\mathbf{B}_{\alpha}(g)(z)$ for all $z \in B$. Since $\mathbf{B}_{\alpha}$ is bounded from $L^{p}\left(d V_{\alpha}\right)$ onto $\mathcal{A}^{p}\left(d V_{\alpha}\right)$ for $1<p<\infty$, this gives us $f \in \mathcal{A}^{p}\left(d V_{\alpha}\right)$ for $1<p<\infty$. (For the case $p=1$, we use the operator $\tilde{\mathbf{B}}_{\alpha}$.)

Fix $p, 1 \leq p<\infty$. Define

$$
\mathcal{B}_{N}=\left\{f \in \mathcal{H}(B) \text { with } \frac{\partial^{N} f}{\partial z_{1}^{k_{1}} \cdots \partial z_{n}^{k_{n}}} \in \mathcal{A}^{p}\left(d V_{\alpha+p N}\right) \text { for all }|\mathbf{k}|=N\right\} \text {. }
$$

It is easy to see that $\mathcal{B}_{N}$ is a Banach space under the norm

$$
\|f\|_{\mathcal{B}_{N}}=\sum_{|\mathbf{k}|=0}^{N-1}\left|\frac{\partial^{|\mathbf{k}|} f}{\partial z_{1}^{k_{1}} \cdots \partial z_{n}^{k_{n}}}(0)\right|+\sum_{|\mathbf{k}|=N}\left\|\frac{\partial^{N} f}{\partial z_{1}^{k_{1}} \cdots \partial z_{n}^{k_{n}}}\right\|_{L^{p}\left(d V_{\alpha+p N}\right)}
$$

If a sequence $\left\{f_{k}\right\}$ converges to $f_{0}$ in $\mathcal{B}_{N}$, then we know that $f_{k} \rightarrow f_{0}$ uniformly on compact subsets of $B$. Now let us prove the estimate (1.4). By (1.5) and Corollary 1.6, we know that $\|f\|_{\mathcal{B}_{N}} \leq C\|f\|_{\mathcal{A}^{p}\left(d V_{\alpha}\right)}$. Next, let $\mathbf{I}: \mathcal{B}_{N} \rightarrow \mathcal{A}^{p}\left(d V_{\alpha}\right)$ be the identity operator. If $\left\|\mathbf{I}\left(f_{k}\right)-F\right\|_{L^{p}\left(d V_{\alpha}\right)} \rightarrow 0$ and $\left\|f_{k}-f_{0}\right\|_{\mathcal{B}_{N}} \rightarrow 0$ as $k \rightarrow \infty$, then $f_{k} \rightarrow F$ uniformly on compact subsets of $B$. Henc, $\mathbf{I}\left(f_{0}\right)=F$. By the closed graph theorem, we know that there exists a constant $C$ such that $\|f\|_{\mathcal{A}^{p}\left(d V_{\alpha}\right)} \leq C\|f\|_{\mathcal{B}_{N}}$. The proof of the theorem is therefore complete. 
We finish this section by considering the case of the polydisc. Let

$$
\mathbb{D}^{n}=\left\{z=\left(z_{1}, \ldots, z_{n}\right) \in \mathbb{C}^{n}:\left|z_{j}\right|<1, j=1, \ldots, n\right\}
$$

be the polydisc in $\mathbb{C}^{n}$ and let $\mathcal{H}\left(\mathbb{D}^{n}\right)$ be the class of all holomorphic functions $f$ defined on $\mathbb{D}^{n}$. Let $\vec{\alpha}=\left(\alpha_{1}, \ldots, \alpha_{n}\right)$ with $\alpha_{j}>-1$ for $j=1, \ldots, n$. The space $L^{p}\left(d V_{\vec{\alpha}}\right)$ consists of all Lebesgue measurable functions defined on $\mathbb{D}^{n}$ satisfying

$$
\|f\|_{L^{p}\left(d V_{\vec{\alpha}}\right)}^{p}=\int_{\mathbb{D}^{n}}|f(z)|^{p} \prod_{j=1}^{n}\left(1-\left|z_{j}\right|^{2}\right)^{\alpha_{j}} d V\left(z_{j}\right)<\infty .
$$

Here $d V\left(z_{j}\right)$ is the normalized volume measure on the unit disc $\mathbb{D}$, i.e.,

$$
\int_{\mathbb{D}} d V\left(z_{j}\right)=\frac{1}{\pi} \int_{0}^{1} \int_{0}^{2 \pi} r d r d \theta_{j}=1 .
$$

Now the weighted Bergman space $\mathcal{A}^{p}\left(d V_{\vec{\alpha}}\right)$ is the intersection of $L^{p}\left(d V_{\vec{\alpha}}\right)$ and $\mathcal{H}\left(\mathbb{D}^{n}\right)$.

From computations in this section, it is easy to see that the kernel $B_{\vec{\alpha}}(z, w)$ for the weighted Bergman projection $\mathbf{B}_{\vec{\alpha}}: L^{2}\left(d V_{\vec{\alpha}}\right) \rightarrow \mathcal{A}^{2}\left(d V_{\vec{\alpha}}\right)$ is

$$
B_{\vec{\alpha}}(z, w)=\prod_{j=1}^{n} \frac{\left(\alpha_{j}+1\right)}{\left(1-z_{j} \bar{w}_{j}\right)^{\alpha_{j}+2}} .
$$

It can be shown that the operator $\mathbf{B}_{\vec{\alpha}}$ can be extended as a bounded operator from $L^{p}\left(d V_{\vec{\alpha}}\right)$ onto $\mathcal{A}^{p}\left(d V_{\vec{\alpha}}\right)$ giving the following theorem:

THEOREM 1.8. Let $N$ be a fixed positive integer and let $\mathbf{k}=\left(k_{1}, \ldots, k_{n}\right)$ $\in\left(\mathbb{Z}_{+}\right)^{n}$. Let $f$ be a holomorphic function defined on the polydisc $\mathbb{D}^{n}$ in $\mathbb{C}^{n}$. Then for $\vec{\alpha}=\left(\alpha_{1}, \ldots, \alpha_{n}\right), f \in \mathcal{A}^{p}\left(d V_{\vec{\alpha}}\right)$ if and only if

$$
\left[\prod_{j=1}^{n}\left(1-\left|z_{j}\right|^{2}\right)^{k_{j}}\right] \frac{\partial^{\mid \mathbf{k}} f}{\partial z_{1}^{k_{1}} \cdots \partial z_{n}^{k_{n}}}(z) \in L^{p}\left(d V_{\vec{\alpha}}\right)
$$

for $1 \leq p<\infty, \alpha_{j}>-1, j=1, \ldots, n$. Moreover

(1.5) $\|f\|_{\mathcal{A}^{p}\left(d V_{\vec{\alpha}}\right)} \approx\left(\sum_{|\mathbf{k}|=0}^{N-1}\left|\frac{\partial^{N} f}{\partial z_{1}^{k_{1}} \cdots \partial z_{n}^{k_{n}}}(0)\right|\right.$

$$
\left.+\sum_{|\mathbf{k}|=N}\left\|\left[\prod_{j=1}^{n}\left(1-\left|z_{j}\right|^{2}\right)^{k_{j}}\right] \frac{\partial^{N} f}{\partial z_{1}^{k_{1}} \cdots \partial z_{n}^{k_{n}}}\right\|_{L^{p}\left(d V_{\vec{\alpha}}\right)}\right)
$$




\section{$\S 2$. The Cesàro operator}

In this section we study the Cesàro operator for the polydisc and ball. We start with the polydisc. Let $f$ be a holomorphic function defined on the polydisc $\mathbb{D}^{n}$. It follows that

$$
f=\sum_{|\mathbf{k}|=0}^{\infty} a_{\mathbf{k}} z^{\mathbf{k}}=\sum_{k_{1}+\cdots+k_{n}=0}^{\infty} a_{k_{1} k_{2} \cdots k_{n}} z_{1}^{k_{1}} z_{2}^{k_{2}} \cdots z_{n}^{k_{n}}
$$

where $\mathbf{k}=\left(k_{1}, \ldots, k_{n}\right) \in\left(\mathbb{Z}_{+}\right)^{n}$. Let $\mathbf{m}=\left(m_{1}, \ldots, m_{n}\right) \in\left(\mathbb{Z}_{+}\right)^{n}$ be another $n$-tuple. We say that $\mathbf{m} \leq \mathbf{k}$ if and only if $m_{j} \leq k_{j}$ for $1 \leq j \leq n$. The Cesàro operator $\mathcal{C}$ is defined by

$$
\mathcal{C}(f)(z)=\sum_{|\mathbf{k}|=0}^{\infty}\left(\frac{1}{\left(k_{1}+1\right) \cdots\left(k_{n}+1\right)} \sum_{\mathbf{m} \leq \mathbf{k}} a_{\mathbf{m}}\right) z^{\mathbf{k}} .
$$

It is easy to see that

$$
\begin{aligned}
\mathcal{C}(f)(z) & =\int_{0}^{1} \cdots \int_{0}^{1} \frac{f\left(t_{1} z_{1}, \ldots, t_{n} z_{n}\right)}{\left(1-t_{1} z_{1}\right) \ldots\left(1-t_{n} z_{n}\right)} d t_{1} \cdots d t_{n} \\
& =\int_{Q} \frac{f(t \cdot z)}{\prod_{j=1}^{n}\left(1-t_{j} z_{j}\right)} d t
\end{aligned}
$$

where $Q=[0,1]^{n}$ and $d t=d t_{1} \cdots d t_{n}$.

In preparation for the proof of Theorem 2.4, we record some preliminary lemmas. The proof of the following lemma is an easy consequence of the plurisubharmonicity of the function $|f|^{p}$.

LEMMA 2.1. Let $1 \leq p<\infty$ and $\vec{\alpha}=\left(\alpha_{1}, \ldots, \alpha_{n}\right)$ with $\alpha_{j}>-1$ for $j=1, \ldots, n$. Then for each $\ell \in\{1, \cdots, n\}$, there exists a universal constant $C_{\ell}$ such that

$$
\|f\|_{\mathcal{A}^{p}\left(d V_{\vec{\alpha}}\right)} \leq C_{\ell}\left\|z_{\ell} f\right\|_{\mathcal{A}^{p}\left(d V_{\vec{\alpha}}\right)}
$$

for every $f \in \mathcal{A}^{p}\left(d V_{\vec{\alpha}}\right)$.

The next lemma is just an $n$-fold version of a result of Duren [D, page $65]$. 
LEMMA 2.2. If $s_{j}>1$ and $0 \leq r_{j}<1$ for $j=1, \ldots, n$, then there is a constant $\gamma$ depending only on $s_{j}, j=1, \ldots, n$, such that

$$
\int_{[-\pi, \pi]^{n}} \prod_{j=1}^{n}\left|1-r_{j} e^{i \theta_{j}}\right|^{-s_{j}} d \theta \leq \gamma \cdot \prod_{j=1}^{n}\left(1-r_{j}\right)^{-s_{j}+1}
$$

where $[-\pi, \pi]^{n}=[-\pi, \pi] \times \cdots \times[-\pi, \pi]$ and $d \theta=\prod_{j=1}^{n} d \theta_{j}$.

The following lemma was first proved by Hardy-Littlewood [HL, pp. 412 and 414] in the case $n=1$. It is not difficult to generalize their result to higher dimensional cases by taking the limit of the sequence of partial sums of the power series expansion of the holomorphic function $f$.

Lemma 2.3. Let $0<p<1,1<q<\infty$ and $0<r_{j}<1$ for $j=$ $1, \ldots, n$. Then there exists two universal constants $C_{1}$ and $C_{2}$ such that

$$
\begin{aligned}
& \int_{[-\pi, \pi]^{n}} \sup _{0 \leq t_{j}<1,1 \leq j \leq n} \mid f(\left.t_{1} r_{1} e^{i \theta_{1}}, \ldots, t_{n} r_{n} e^{i \theta_{n}}\right)\left.\right|^{p} d \theta \\
& \leq C_{1} \int_{[-\pi, \pi]^{n}}\left|f\left(r_{1} e^{i \theta_{1}}, \ldots, r_{n} e^{i \theta_{n}}\right)\right|^{p} d \theta
\end{aligned}
$$

and

$$
\begin{aligned}
& \int_{Q}\left\{\int_{[-\pi, \pi]^{n}} \mid f\left(t_{1} r_{1} e^{i \theta_{1}}, \ldots,\right.\right.\left.\left.t_{n} r_{n} e^{i \theta_{n}}\right)\left.\right|^{p q} d \theta\right\}^{\frac{1}{q}} \prod_{j=1}^{n}\left(1-t_{j}\right)^{-\frac{1}{q}} d t \\
& \leq C_{2} \int_{[-\pi, \pi]^{n}}\left|f\left(r_{1} e^{i \theta_{1}}, \ldots, r_{n} e^{i \theta_{n}}\right)\right|^{p} d \theta
\end{aligned}
$$

for all holomorphic functions $f$ defined on $\mathbb{D}^{n}$.

Now we are in a position to prove the first of our two theorems on the Cesàro operator.

TheOREM 2.4. Let $\vec{\alpha}=\left(\alpha_{1}, \ldots, \alpha_{n}\right)$ with $\alpha_{j}>-1$ for $j=1, \ldots, n$. Then the Cesàro operator $\mathcal{C}$ is bounded on $\mathcal{A}^{p}\left(d V_{\vec{\alpha}}\right)$ for $0<p<\infty$.

Proof. We have to split the proof of this theorem into two cases. 
Case $1.1 \leq p<\infty$. Suppose that $f \in \mathcal{A}^{p}\left(d V_{\vec{\alpha}}\right)$ and let $F=\mathcal{C}(f)$. By direct computation, we obtain

$$
\begin{aligned}
& z_{1} \cdots z_{n} \frac{\partial^{n} F(z)}{\partial z_{1} \cdots \partial z_{n}}=\frac{f\left(z_{1}, \ldots, z_{n}\right)}{\left(1-z_{1}\right) \cdots\left(1-z_{n}\right)} \\
+ & \sum_{q=1}^{n-1}(-1)^{q} \times \\
& \sum_{1 \leq j_{1}<\cdots<j_{q} \leq n} \int_{[0,1]^{q}} \frac{f\left(z_{1}, \ldots, t_{j_{1}} z_{j_{1}}, \ldots, t_{j_{q}} z_{j_{q}}, \ldots, z_{n}\right) d t_{j_{1}} \cdots d t_{j_{q}}}{\left(1-z_{1}\right) \cdots\left(1-t_{j_{1}} z_{j_{1}}\right) \cdots\left(1-t_{j_{q}} z_{j_{q}}\right) \cdots\left(1-z_{n}\right)} \\
+ & (-1)^{n} \int_{[0,1]^{n}} \frac{f\left(t_{1} z_{1}, \ldots, t_{n} z_{n}\right)}{\left(1-t_{1} z_{1}\right) \cdots\left(1-t_{n} z_{n}\right)} d t_{1} \cdots d t_{n} .
\end{aligned}
$$

It is easy to see that the first term on the right hand side of (2.1) satisfies the following estimate.

$$
\begin{aligned}
& \int_{\mathbb{D}^{n}}\left|\prod_{j=1}^{n}\left(1-\left|z_{j}\right|^{2}\right) \frac{f\left(z_{1}, \ldots, z_{n}\right)}{\left(1-z_{j}\right)}\right|^{p} \prod_{j=1}^{n}\left(1-\left|z_{j}\right|^{2}\right)^{\alpha_{j}} d V(z) \\
\leq & \int_{\mathbb{D}^{n}}\left|f\left(z_{1}, \ldots, z_{n}\right)\right|^{p} \prod_{j=1}^{n}\left(1+\left|z_{j}\right|\right)^{p}\left(1-\left|z_{j}\right|^{2}\right)^{\alpha_{j}} d V(z) \\
\leq & 2^{n p} \int_{\mathbb{D}^{n}}|f(z)|^{p} \prod_{j=1}^{n}\left(1-\left|z_{j}\right|^{2}\right)^{\alpha_{j}} d V(z) .
\end{aligned}
$$

For the last term on the right hand side of (2.1), we have

$$
\begin{aligned}
& \left\|\prod_{j=1}^{n}\left(1-\left|z_{j}\right|^{2}\right) \int_{Q} \frac{f(t \cdot z)}{\left(1-t_{1} z_{1}\right) \cdots\left(1-t_{n} z_{n}\right)} d t\right\|_{L^{p}\left(d V_{\vec{\alpha}}\right)} \|_{Q}\left\{\int_{\mathbb{D}^{n}}\left[\prod_{j=1}^{n} \frac{\left(1-\left|z_{j}\right|^{2}\right)}{\left|1-t_{j} z_{j}\right|}|f(t \cdot z)|\right]_{j=1}^{p}\left(1-\left|z_{j}\right|^{2}\right)^{\alpha_{j}} d V(z)\right\}^{\frac{1}{p}} d t \\
\leq & 2^{n} \int_{Q}\left\{\int_{\mathbb{D}^{n}}\left|f\left(t_{1} z_{1}, \ldots, t_{n} z_{n}\right)\right|^{p} \prod_{j=1}^{n}\left(1-\left|z_{j}\right|^{2}\right)^{\alpha_{j}} d V(z)\right\}^{\frac{1}{p}} d t \\
\leq & 2^{n} \int_{Q}\left\{\int_{\mathbb{D}^{n}}\left|f\left(z_{1}, \ldots, z_{n}\right)\right|^{p} \prod_{j=1}^{n}\left(1-\left|z_{j}\right|^{2}\right)^{\alpha_{j}} d V(z)\right\}^{\frac{1}{p}} d t
\end{aligned}
$$




$$
=2^{n}\|f\|_{\mathcal{A}^{p}\left(d V_{\vec{\alpha}}\right)}
$$

Let $J=\left(j_{1}, \ldots, j_{q}\right)$ with $1 \leq j_{1}<\cdots<j_{q} \leq n$. For terms in between on the right hand side of $(2.1)$, we have

$$
\begin{aligned}
& \| \prod_{j=1}^{n}\left(1-\left|z_{j}\right|^{2}\right) \times \\
& \times \int_{[0,1]^{q}} \frac{f\left(z_{1}, \ldots, t_{j_{1}} z_{j_{1}} \ldots, t_{j_{q}} z_{j_{q}}, \ldots, z_{n}\right)}{\left(1-z_{1}\right) \cdots\left(1-t_{j_{1}} z_{j_{n}}\right) \cdots\left(1-t_{j_{q}} z_{j_{q}}\right), \cdots\left(1-z_{n}\right)} d t \|_{L^{p}\left(d V_{\vec{\alpha}}\right)} \\
& \leq \int_{[0,1]^{q}}\left\{\int_{\mathbb{D}^{n}}\left[\prod_{j \notin J} \frac{\left(1-\left|z_{j}\right|^{2}\right)}{\left|1-z_{j}\right|} \prod_{j \in J} \frac{\left(1-\left|z_{j}\right|^{2}\right)}{\left|1-t_{j} z_{j}\right|}|f(t \cdot z)|\right]^{p} \times\right. \\
& \left.\times \prod_{j=1}^{n}\left(1-\left|z_{j}\right|^{2}\right)^{\alpha_{j}} d V\left(z_{j}\right)\right\}^{\frac{1}{p}} d t \\
& \leq 2^{n} \int_{[0,1]^{q}}\left\{\int_{\mathbb{D}^{n}}\left|f\left(z_{1}, \ldots, t_{j_{1}} z_{j_{1}}, \ldots, t_{j_{q}} z_{j_{q}}, \ldots, z_{n}\right)\right|^{p} \times\right. \\
& \left.\times \prod_{j=1}^{n}\left(1-\left|z_{j}\right|^{2}\right)^{\alpha_{j}} d V\left(z_{j}\right)\right\}^{\frac{1}{p}} d t \\
& \leq 2^{n} \int_{[0,1]^{q}}\left\{\int_{\mathbb{D}^{n}}\left|f\left(z_{1}, \ldots, z_{n}\right)\right|^{p} \prod_{j=1}^{n}\left(1-\left|z_{j}\right|^{2}\right)^{\alpha_{j}} d V\left(z_{j}\right)\right\}^{\frac{1}{p}} d t \\
& =2^{n}\|f\|_{\mathcal{A}^{p}\left(d V_{\vec{\alpha}}\right)} .
\end{aligned}
$$

Here we use the Minkowski integral inequality and the monotonicity of the function $U\left(t_{1}, \ldots, t_{n}\right)=\int_{0}^{2 \pi} \cdots \int_{0}^{2 \pi}\left|f\left(t_{1} r_{1} e^{i \theta_{1}}, \ldots, t_{n} r_{n} e^{i \theta_{n}}\right)\right|^{p} d \theta_{1} \cdots d \theta_{n}$. Combining the above computations, we obtain

$$
\left\|\prod_{j=1}^{n}\left(1-\left|z_{j}\right|^{2}\right)\left[z_{1} \cdots z_{n} \frac{\partial^{n} F(z)}{\partial z_{1} \cdots \partial z_{n}}\right]\right\|_{L^{p}\left(d V_{\vec{\alpha}}\right)} \leq 2^{2 n}\|f\|_{\mathcal{A}^{p}\left(d V_{\vec{\alpha}}\right)} .
$$

But the left hand side of the above inequality is equivalent to

$$
z_{1} \cdots z_{n} \frac{\partial^{n} F(z)}{\partial z_{1} \cdots \partial z_{n}} \in \mathcal{A}^{p}\left(d V_{\vec{\alpha}+\mathbf{p}}\right)
$$


with $\vec{\alpha}+\mathbf{p}=\left(\alpha_{1}+p, \ldots, \alpha_{n}+p\right)$. By Lemma 2.1, there exists a universal constant $C$ such that

$$
\begin{aligned}
& \left\|\prod_{j=1}^{n}\left(1-\left|z_{j}\right|^{2}\right) \frac{\partial^{n} F(z)}{\partial z_{1} \cdots \partial z_{n}}\right\|_{L^{p}\left(d V_{\vec{\alpha}}\right)} \leq C^{\prime}\left\|z_{1} \cdots z_{n} \frac{\partial^{n} F(z)}{\partial z_{1} \cdots \partial z_{n}}\right\|_{\mathcal{A}^{p}\left(d V_{\vec{\alpha}+\mathbf{p}}\right)} \\
= & \left\|\frac{\partial^{n} F(z)}{\partial z_{1} \cdots \partial z_{n}}\right\|_{\mathcal{A}^{p}\left(d V_{\vec{\alpha}+\mathbf{p}}\right)} \| \\
= & C^{\prime}\left\|\prod_{j=1}^{n}\left(1-\left|z_{j}\right|^{2}\right) z_{1} \cdots z_{n} \frac{\partial^{n} F(z)}{\partial z_{1} \cdots \partial z_{n}}\right\|_{L^{p}\left(d V_{\vec{\alpha}}\right)} \cdot
\end{aligned}
$$

It follows that

$$
\left\|\prod_{j=1}^{n}\left(1-\left|z_{j}\right|^{2}\right) \frac{\partial^{n} F(z)}{\partial z_{1} \cdots \partial z_{n}}\right\|_{L^{p}\left(d V_{\vec{\alpha}}\right)} \leq c\|f\|_{\mathcal{A}^{p}\left(d V_{\vec{\alpha}}\right)} .
$$

Since $F(0)=f(0)$, by Theorem 1.8 , we have

$$
\begin{aligned}
\|F\|_{\mathcal{A}^{p}\left(d V_{\vec{\alpha}}\right)} & =\|\mathcal{C}(f)\|_{\mathcal{A}^{p}\left(d V_{\vec{\alpha}}\right)} \\
& \leq c\left(|f(0)|+\left\|\prod_{j=1}^{n}\left(1-\left|z_{j}\right|^{2}\right) \frac{\partial^{n} F(z)}{\partial z_{1} \cdots \partial z_{n}}\right\|_{L^{p}\left(d V_{\vec{\alpha}}\right)}\right) \\
& \leq c^{\prime}\|f\|_{\mathcal{A}^{p}\left(d V_{\vec{\alpha}}\right)} .
\end{aligned}
$$

Here $c$ and $c^{\prime}$ are universal constants depending on $p$ and $\alpha$ only.

Case 2. $0<p<1$. Without loss of generality, we may just assume $n=2$. Let $f \in \mathcal{A}^{p}\left(d V_{\vec{\alpha}}\right)$ and $F=\mathcal{C}(f)$. Suppose that $1<q<\frac{1}{1-p}$ and $q^{\prime}$ is the conjugate exponent of $q$, i.e., $\frac{1}{q}+\frac{1}{q^{\prime}}=1$. Then by Lemma 2.2 and Hölder's inequality, we have

$$
\begin{aligned}
& \int_{[-\pi, \pi]^{2}}\left|\frac{f\left(t_{1} r_{1} e^{i \theta_{1}}, t_{2} r_{2} e^{i \theta_{2}}\right)}{\left(1-t_{1} r_{1} e^{i \theta_{1}}\right)\left(1-t_{2} r_{2} e^{i \theta_{2}}\right)}\right|^{p} d \theta_{1} d \theta_{2} \\
\leq & {\left[\int_{[-\pi, \pi]^{2}}\left|\frac{1}{\left(1-t_{1} r_{1} e^{i \theta_{1}}\right)\left(1-t_{2} r_{2} e^{i \theta_{2}}\right)}\right|^{p q^{\prime}} d \theta_{1} d \theta_{2}\right]^{\frac{1}{q^{\prime}}} \times }
\end{aligned}
$$




$$
\begin{aligned}
& \times\left[\int_{[-\pi, \pi]^{2}}\left|f\left(t_{1} r_{1} e^{i \theta_{1}}, t_{2} r_{2} e^{i \theta_{2}}\right)\right|^{p q} d \theta_{1} d \theta_{2}\right]^{\frac{1}{q}} \\
\leq & C_{\gamma} \prod_{j=1}^{2}\left(1-t_{j}\right)^{\frac{1-p q^{\prime}}{q^{\prime}}}\left[\int_{[-\pi, \pi]^{2}}\left|f\left(t_{1} r_{1} e^{i \theta_{1}}, t_{2} r_{2} e^{i \theta_{2}}\right)\right|^{p q} d \theta_{1} d \theta_{2}\right]^{\frac{1}{q}} .
\end{aligned}
$$

Now let us consider a partition on the unit interval $[0,1]$ with $\lambda_{j}=1-2^{-j}$ and $\lambda_{k}=1-2^{-k}$ for $j, k \in \mathbb{Z}_{+}$. Then we obtain

$$
\int_{[-\pi, \pi]^{2}}\left|F\left(r_{1} e^{i \theta_{1}}, r_{2} e^{i \theta_{2}}\right)\right|^{p} d \theta_{1} d \theta_{2}
$$

$$
\leq \int_{[-\pi, \pi]^{2}}\left\{\int_{[0,1]^{2}}\left|\frac{f\left(t_{1} r_{1} e^{i \theta_{1}}, t_{2} r_{2} e^{i \theta_{2}}\right)}{\left(1-t_{1} r_{1} e^{i \theta_{1}}\right)\left(1-t_{2} r_{2} e^{i \theta_{2}}\right)}\right| d t_{1} d t_{2}\right\}^{p} d \theta_{1} d \theta_{2}
$$$$
\leq \sum_{j, k=1}^{\infty} \int_{[-\pi, \pi]^{2}}\left\{\int_{\lambda_{k-1}}^{\lambda_{k}} \int_{\lambda_{j-1}}^{\lambda_{j}}\left|\frac{f\left(t_{1} r_{1} e^{i \theta_{1}}, t_{2} r_{2} e^{i \theta_{2}}\right)}{\left(1-t_{1} r_{1} e^{i \theta_{1}}\right)\left(1-t_{2} r_{2} e^{i \theta_{2}}\right)}\right| d t_{1} d t_{2}\right\}^{p} d \theta_{1} d \theta_{2}
$$

$$
\begin{aligned}
\leq \sum_{j, k=1}^{\infty} & \frac{1}{2^{(j+k) p}} \times \\
& \times \int_{[-\pi, \pi]^{2}}\left\{\sup _{0 \leq t_{1} \leq \lambda_{j}, 0 \leq t_{2} \leq \lambda_{k}}\left|\frac{f\left(t_{1} r_{1} e^{i \theta_{1}}, t_{2} r_{2} e^{i \theta_{2}}\right)}{\left(1-t_{1} r_{1} e^{i \theta_{1}}\right)\left(1-t_{2} r_{2} e^{i \theta_{2}}\right)}\right|^{p}\right\} d \theta_{1} d \theta_{2} .
\end{aligned}
$$

Next let us analyse the last line of (2.3). By the Hardy-Littlewood inequality, we know that

$$
\begin{aligned}
& \int_{[-\pi, \pi]^{2}}\left\{\sup _{0 \leq t_{1} \leq \lambda_{j}, 0 \leq t_{2} \leq \lambda_{k}}\left|\frac{f\left(t_{1} r_{1} e^{i \theta_{1}}, t_{2} r_{2} e^{i \theta_{2}}\right)}{\left(1-t_{1} r_{1} e^{i \theta_{1}}\right)\left(1-t_{2} r_{2} e^{i \theta_{2}}\right)}\right|^{p}\right\} d \theta_{1} d \theta_{2} \\
& \leq \int_{[-\pi, \pi]^{2}}\left\{\sup _{0 \leq t_{1}<\lambda_{j}, 0 \leq t_{2}<\lambda_{k}}\left|\frac{f\left(t_{1} r_{1} e^{i \theta_{1}}, t_{2} r_{2} e^{i \theta_{2}}\right)}{\left(1-t_{1} r_{1} e^{i \theta_{1}}\right)\left(1-t_{2} r_{2} e^{i \theta_{2}}\right)}\right|^{p}+\right. \\
& \left.+\left|\frac{f\left(\lambda_{j} r_{1} e^{i \theta_{1}}, \lambda_{k} r_{2} e^{i \theta_{2}}\right)}{\left(1-\lambda_{j} r_{1} e^{i \theta_{1}}\right)\left(1-\lambda_{k} r_{2} e^{i \theta_{2}}\right)}\right|^{p}\right\} d \theta_{1} d \theta_{2} \\
& \leq\left(C_{1}+1\right) \int_{[-\pi, \pi]^{2}}\left|\frac{f\left(\lambda_{j} r_{1} e^{i \theta_{1}}, \lambda_{k} r_{2} e^{i \theta_{2}}\right)}{\left(1-\lambda_{j} r_{1} e^{i \theta_{1}}\right)\left(1-\lambda_{k} r_{2} e^{i \theta_{2}}\right)}\right|^{p} d \theta_{1} d \theta_{2} .
\end{aligned}
$$


For $\lambda_{j-1} \leq t_{1}<1$ and $\lambda_{k-1} \leq t_{2}<1$, we use a similar trick to obtain

$$
\begin{aligned}
& \int_{[-\pi, \pi]^{2}}\left|\frac{f\left(\lambda_{j-1} r_{1} e^{i \theta_{1}}, \lambda_{k-1} r_{2} e^{i \theta_{2}}\right)}{\left(1-\lambda_{j-1} r_{1} e^{i \theta_{1}}\right)\left(1-\lambda_{k-1} r_{2} e^{i \theta_{2}}\right)}\right|^{p} d \theta_{1} d \theta_{2} \\
\leq & \int_{[-\pi, \pi]^{2}}\left\{\sup _{0 \leq \rho_{1}<t_{1}, 0 \leq \rho_{2}<t_{2}}\left|\frac{f\left(\rho_{1} r_{1} e^{i \theta_{1}}, \rho_{2} r_{2} e^{i \theta_{2}}\right)}{\left(1-\rho_{1} r_{1} e^{i \theta_{1}}\right)\left(1-\rho_{2} r_{2} e^{i \theta_{2}}\right)}\right|^{p}\right\} d \theta_{1} d \theta_{2} \\
\leq & \left(C_{1}+1\right) \int_{[-\pi, \pi]^{2}}\left|\frac{f\left(t_{1} r_{1} e^{i \theta_{1}}, t_{2} r_{2} e^{i \theta_{2}}\right)}{\left(1-t_{1} r_{1} e^{i \theta_{1}}\right)\left(1-t_{2} r_{2} e^{i \theta_{2}}\right)}\right|^{p} d \theta_{1} d \theta_{2} .
\end{aligned}
$$

Combining (2.4) and (2.5), we get the following

$$
\begin{aligned}
& \sum_{j, k=1}^{\infty} \frac{1}{2^{(j+k) p}} \times \\
& \quad \times \int_{[-\pi, \pi]^{2}}\left\{\sup _{0 \leq t_{1} \leq \lambda_{j}, 0 \leq t_{2} \leq \lambda_{k}}\left|\frac{f\left(t_{1} r_{1} e^{i \theta_{1}}, t_{2} r_{2} e^{i \theta_{2}}\right)}{\left(1-t_{1} r_{1} e^{i \theta_{1}}\right)\left(1-t_{2} r_{2} e^{i \theta_{2}}\right)}\right|^{p}\right\} d \theta_{1} d \theta_{2} \\
& \leq\left(C_{1}+1\right) \sum_{j, k=1}^{\infty} \frac{1}{2^{(j+k) p}} \int_{[-\pi, \pi]^{2}}\left|\frac{f\left(\lambda_{j} r_{1} e^{i \theta_{1}}, \lambda_{k} r_{2} e^{i \theta_{2}}\right)}{\left(1-\lambda_{j} r_{1} e^{i \theta_{1}}\right)\left(1-\lambda_{k} r_{2} e^{i \theta_{2}}\right)}\right|^{p} d \theta_{1} d \theta_{2} .
\end{aligned}
$$

In fact, (2.6) can be estimates as follows:

$$
\begin{aligned}
&(2.6) \leq 2^{2}(\left.C_{1}+1\right) \sum_{j, k=1}^{\infty}\left(1-\lambda_{j}\right)^{p-1}\left(1-\lambda_{k}\right)^{p-1} \times \\
& \times\left\{\int_{[-\pi, \pi]^{2}}\left|\frac{f\left(\lambda_{j} r_{1} e^{i \theta_{1}}, \lambda_{k} r_{2} e^{i \theta_{2}}\right)}{\left(1-\lambda_{j} r_{1} e^{i \theta_{1}}\right)\left(1-\lambda_{k} r_{2} e^{i \theta_{2}}\right)}\right|^{p} d \theta_{1} d \theta_{2}\right\} \times\left(\lambda_{j+1}-\lambda_{j}\right)\left(\lambda_{k+1}-\lambda_{k}\right) \\
& \leq 2^{4}\left(C_{1}+1\right) \sum_{j, k=1}^{\infty}\left(1-\lambda_{j}\right)^{p-1}\left(1-\lambda_{k}\right)^{p-1} \times \\
& \times\left\{\int_{[-\pi, \pi]^{2}}\left|\frac{f\left(\lambda_{j} r_{1} e^{i \theta_{1}}, \lambda_{k} r_{2} e^{i \theta_{2}}\right)}{\left(1-\lambda_{j} r_{1} e^{i \theta_{1}}\right)\left(1-\lambda_{k} r_{2} e^{i \theta_{2}}\right)}\right|^{p} d \theta_{1} d \theta_{2}\right\} \times \\
& \times\left(\lambda_{j}-\lambda_{j-1}\right)\left(\lambda_{k}-\lambda_{k-1}\right)
\end{aligned}
$$




$$
\begin{aligned}
& \leq 2^{4}\left(C_{1}+1\right)^{2} \sum_{j, k=1}^{\infty} \int_{\lambda_{j-1}}^{\lambda_{j}} \int_{\lambda_{k-1}}^{\lambda_{k}} \prod_{\ell=1}^{2}\left(1-t_{\ell}\right)^{p-1} \times \\
& \quad \times\left\{\int_{[-\pi, \pi]^{2}}\left|\frac{f\left(t_{1} r_{1} e^{i \theta_{1}}, t_{2} r_{2} e^{i \theta_{2}}\right)}{\left(1-t_{1} r_{1} e^{i \theta_{1}}\right)\left(1-t_{2} r_{2} e^{i \theta_{2}}\right)}\right|^{p} d \theta_{1} d \theta_{2}\right\} d t_{1} d t_{2} .
\end{aligned}
$$

This is actually bounded by the following:

$$
\begin{aligned}
& C_{\gamma} 2^{4}\left(C_{1}+1\right)^{2} \int_{[0,1]^{2}} \prod_{\ell=1}^{2}\left(1-t_{\ell}\right)^{p-1}\left(1-t_{\ell}\right)^{\frac{1-p q^{\prime}}{q^{\prime}}} \times \\
& \times\left[\int_{[-\pi, \pi]^{2}}\left|f\left(t_{1} r_{1} e^{i \theta_{1}}, t_{2} r_{2} e^{i \theta_{2}}\right)\right|^{p q} d \theta_{1} d \theta_{2}\right]^{\frac{1}{q}} d t_{1} d t_{2} \\
\leq & C_{3} \int_{[0,1]^{2}} \prod_{\ell=1}^{2}\left(1-t_{\ell}\right)^{-\frac{1}{q}}\left[\int_{[-\pi, \pi]^{2}}\left|f\left(t_{1} r_{1} e^{i \theta_{1}}, t_{2} r_{2} e^{i \theta_{2}}\right)\right|^{p q} d \theta_{1} d \theta_{2}\right]^{\frac{1}{q}} d t_{1} d t_{2} \\
\leq & C_{4} \int_{[-\pi, \pi]^{2}}^{\left|f\left(r_{1} e^{i \theta_{1}}, r_{2} e^{i \theta_{2}}\right)\right|^{p} d \theta_{1} d \theta_{2} .}
\end{aligned}
$$

Here we use (2.2) and Lemma 2.3. It follows that

$$
\|\mathcal{C}(f)\|_{\mathcal{A}^{p}\left(d V_{\vec{\alpha}}\right)} \leq C \cdot\|f\|_{\mathcal{A}^{p}\left(d V_{\vec{\alpha}}\right)} .
$$

The proof of this theorem is therefore complete.

We next consider a Cesàro operator on $\mathcal{A}^{p}\left(d V_{\alpha}\right)$, which we define below. Let $f$ be a holomorphic function defined on the unit ball $B$. Assume that $f(z)=\sum_{|\mathbf{k}|=0}^{\infty} a_{\mathbf{k}} z^{\mathbf{k}}$. For $\ell \in \mathbb{Z}_{+}$, let $F_{\ell}(z)=\sum_{|\mathbf{k}|=\ell} a_{\mathbf{k}} z^{\mathbf{k}}$. It follows that $F_{\ell}$ is homogeneous of degree $\ell$, and the power series can be rewritten as the homogeneous expansion as follows:

$$
f(z)=\sum_{\ell=0}^{\infty} F_{\ell}(z)
$$

Now fix a point $\zeta \in \partial B$, then

$$
f(z)=f(\zeta \cdot \xi)=f_{\zeta}(\xi)=\sum_{\ell=0}^{\infty} F_{\ell}(\zeta) \xi^{\ell}
$$


for $\xi \in \mathbb{D}$. It has been shown that the infinite series $\sum_{\ell=0}^{\infty} F_{\ell}(\zeta) \xi^{\ell}$ converges uniformly to $f(z)$ on every compact subset in $\mathbb{D}$ (see Rudin [R, pages 19$22]$ ). It is obvious that $|\xi|=|\zeta \cdot \xi|=|z|=r$. We define the "slice Cesàro operator" as follows:

$$
\mathcal{C}_{s}(f)(z)=\mathcal{C}_{s}\left(f_{\zeta}\right)(\xi)=\sum_{k=0}^{\infty}\left[\frac{1}{k+1} \sum_{\ell=0}^{k} F_{\ell}(\zeta)\right] \xi^{k}
$$

It is easy to see that

$$
\mathcal{C}_{s}(f)(z)=\int_{0}^{1} \frac{f_{\zeta}(t \xi)}{(1-t \xi)} d t
$$

An argument similar to the one above can be used to prove the following theorem:

TheOREM 2.5. Cesàro operator $\mathcal{C}_{s}$ is bounded on $\mathcal{A}^{p}\left(d V_{\alpha}\right)$ for $0<p<$ $\infty$

\section{REFERENCES}

[BL] S. Bell and E. Ligocka, A simplification and extension of Fefferman's theorem on biholomorphic mappings, Invent. Math., 57 (1980), 283-289.

[BG] A. Bonami and S. Grellier, Weighted Bergman projections in domains of finite type in $\mathbb{C}^{2}$, Contemporary Math., 189 (1995), 65-80.

[BCG] A. Bonami, D.C. Chang and S. Grellier, Commutation Properties and Lipschitz estimates for the Bergman and Szegö projections, Math. Zeit., 223 (1996), 275-302.

[Ca] D. Catlin, Subelliptic estimates for the $\bar{\partial}-$ Neumann problem on pseudoconvex domains, Ann. of Math., 126 (1987), 131-191.

[CL] D.C. Chang and B.Q. Li, Sobolev and Lipschitz Estimates for weighted Bergman projections, Nagoya Mathematical Journal, 147 (1997), 147-178.

[CNS] D.C. Chang, A. Nagel and E.M. Stein, Estimates for the $\bar{\partial}$-Neumann problem in pseudoconvex domains of finite type in $\mathbf{C}^{2}$, Acta Mathemtica, 169 (1992), 153-228.

[D] P.L. Duren, Theory of $H^{p}$ Spaces, Academic Press, New York, 1970.

[DS] A.E. Djrbashian and F.A. Shamoian, Topics in the Theory of $A_{\alpha}^{p}$ Spaces, Teubner Verlagsgellschaft, Leibzig, 1988.

[F] C.L. Fefferman, The Bergman kernel and biholomorphic mappings of pseudoconvex domains, Invent. Math., 26 (1974), 1-65.

[Fo] F. Forelli, Measures whose Poisson integrals are plurisubharmonic, Illinois J. Math., 18 (1974), 373-388. 
[HL] G.H. Hardy and J.E. Littlewoood, Some properties of fractional integrals II, Math. Zeit., 34 (1932), 403-439.

[K] S.G. Krantz, Function Theory of Several Complex Variables (2nd edition), Wadsworth \& Brooks/Cole, Pacific Grove, California, 1992.

[M] J. Miao, The Cesáro operator is bounded on $H^{p}$ for $0<p \leq 1$, Proc. Amer. Math. Soc., 116 (1992), 1077-1079.

[R] W. Rudin, Function Theory on the Unit Ball of $\mathbb{C}^{n}$, Springer-Verlag, Berlin·New York·Heidelberg, 1980.

[Si] A.G. Siskakis, The Cesáro operator is bounded on $H^{1}$, Proc. Amer. Math. Soc., 110 (1990), 461-462.

[T] E.C. Titchmarsh, The Theory of Functions, Oxford University Press, London, 1968.

[Z] K. Zhu, Operator Theory in Function Spaces, Marcel Dekker, Inc., New York. Basel, 1990.

George Benke

Department of Mathematics

Georgetown University

Washington D.C.

20057, U.S.A.

benke@math.georgetown.edu

Der-Chen Chang

Department of Mathematics

Georgetown University

Washington D.C.

20057, U.S.A.

chang@math.georgetown.edu 